\title{
Single phase transformerless inverter topologies for grid-tied photovoltaic system: A review
}

\author{
Monirul Islam ${ }^{\text {a,*, Saad Mekhilef }}{ }^{\text {a,*, }}$, Mahamudul Hasan ${ }^{\text {b }}$ \\ ${ }^{a}$ Power Electronics and Renewable Energy Research Laboratory (PEARL), Department of Electrical Engineering Faculty of Engineering, University of Malaya, \\ Kuala Lumpur 50603, Malaysia
}

${ }^{\mathrm{b}}$ Department of Mechanical Engineering, University of Malaya, Kuala Lumpur 50603, Malaysia

\section{A R T I C L E I N F O}

Article history:

Received 10 October 2013

Received in revised form

12 December 2014

4015

Available online 31 January 2015

Keywords:

Common mode voltage

Grid connected

Leakage current

Renewable ener

Photovoltaic

ransformerless inverte

\section{A B S T R A C T}

Grid-tied inverters are the key components of distributed generation system because of their function as an effective interface between renewable energy sources and utility. Recently, there has been an increasing interest in the use of transformerless inverter for low-voltage single-phase grid-tied photovoltaic (PV) system due to higher efficiency, lower cost, smaller size and weight when compared to the ones with transformer. However, the leakage current issues of transformerless inverter, which depends on the topology structure and modulation scheme, have to be addressed very carefully. This review focuses on the transformerless topologies, which are classified into three basic groups based on the decoupling method and leakage current characteristics. Different topologies under the three classes are presented, compared and evaluated based on leakage current, component ratings, advantages, and disadvantages. An examination of demand for the inverter, the utility grid, and the PV module are presented. A performance comparison in MATLAB/Simulink environment is done among different topologies. Also an analysis has been presented to select a better topology. Finally, based on the analysis and simulation results, a comparison table has been presented. Furthermore, some important experimental parameters have been summarized.

c 2015 Elsevier Ltd. All rights reserved.

\section{Contents}

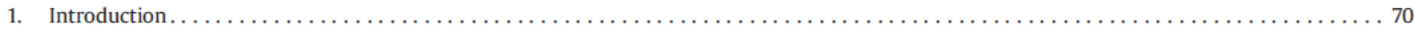

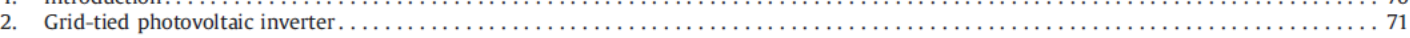

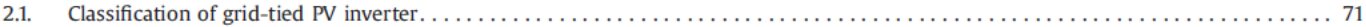

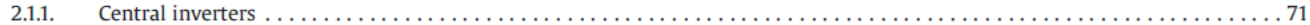

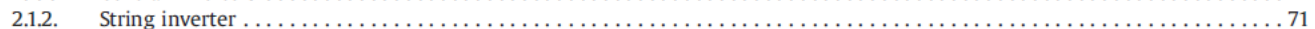

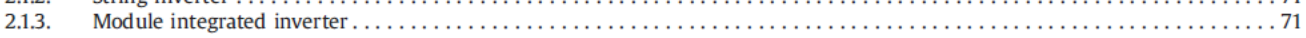

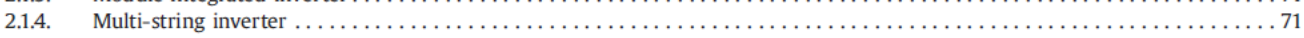

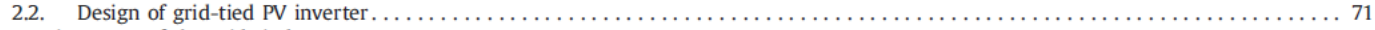

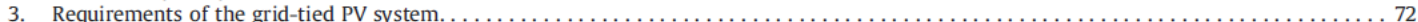

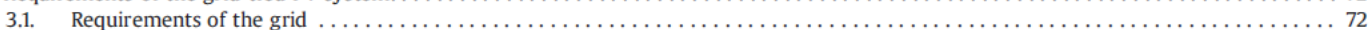

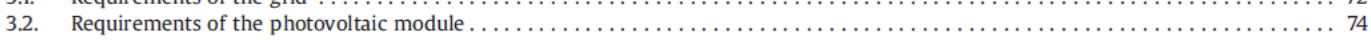

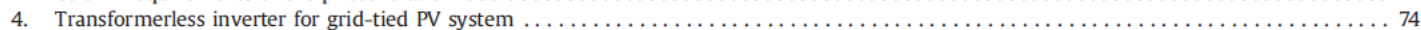

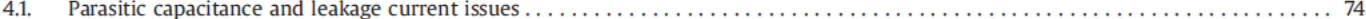

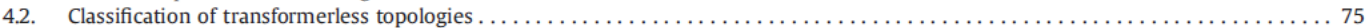

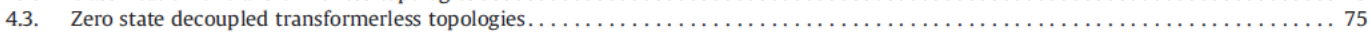

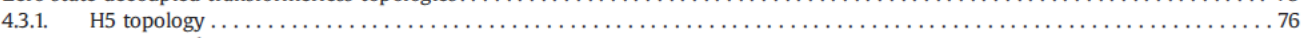

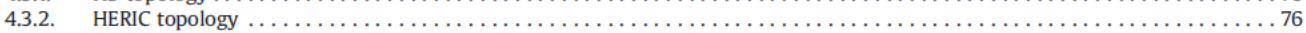

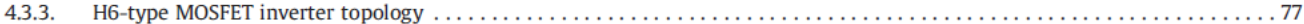

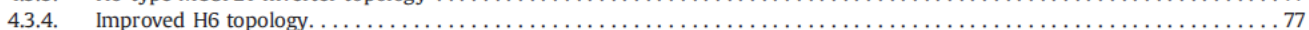

\footnotetext{
${ }^{*}$ Corresponding authors.

E-mail addresses: monirO4eee@yahoo.com (M. Islam), saad@um.edu.my (S. Mekhilef).

http://dx.doi.org/10.1016/j.rser.2015.01.009

1364-0321/\% 2015 Elsevier Ltd. All rights reserved.
} 


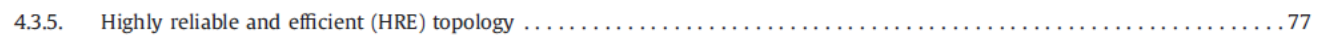

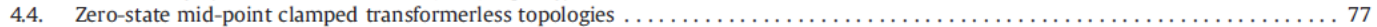

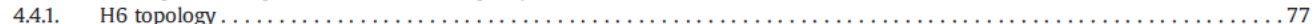

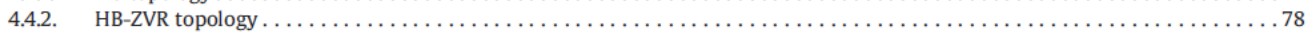

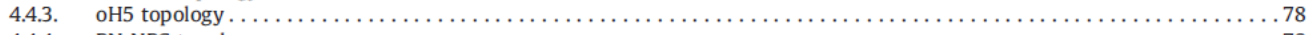

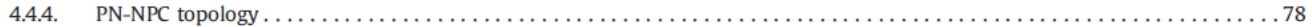

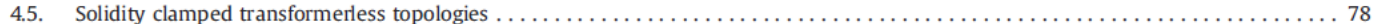

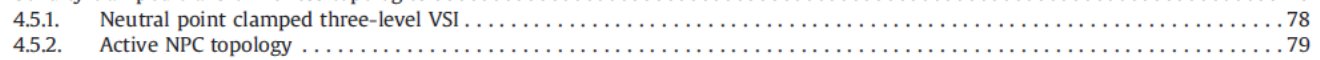

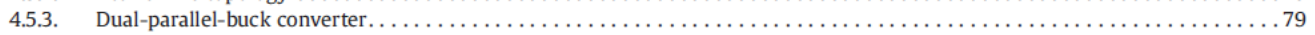

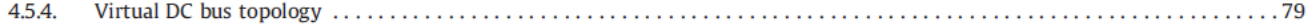

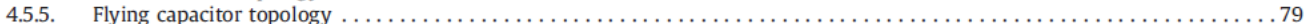

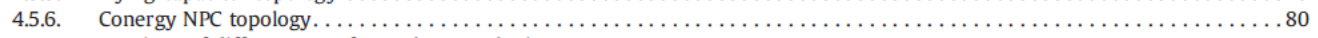

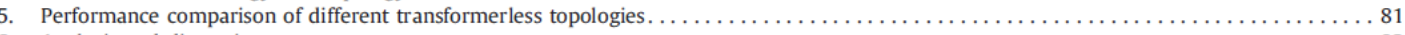

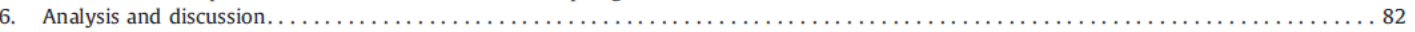

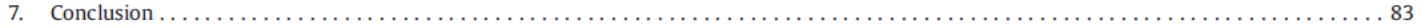

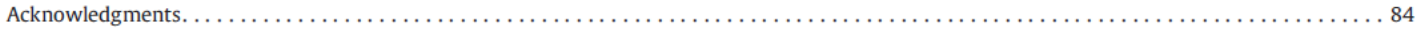

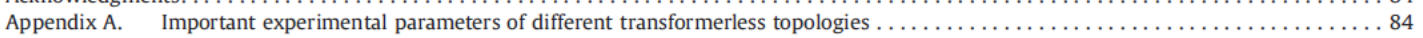

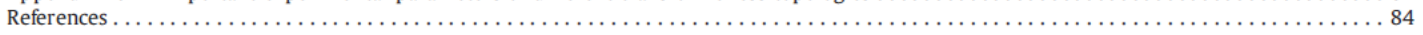

\section{Introduction}

The conventional generation systems such as coal fired, gas and nuclear power as well as hydroelectric dams are centralized and often require electricity to be transmitted over long distances. The security and stability of the conventional electrical power system are under threat due to a number of blackouts caused by chain failure [1] and electric grid ruptures caused by extreme weather [2]. In contrast, distributed energy resources such as solar power, wind power, biomass, and biogas are decentralized, modular and more flexible. As well, these energy sources have the advantage that the power is produced in close proximity to where it is consumed $[3,4]$. This way the losses due to transmission lines can be reduced. In addition, the constant consumption of fossil fuels is leading to energy crisis and increasing environmental pollution problems. Therefore, the distributed energy resources, particularly PV and wind power basis [5], have achieved great response in current years to meet the world energy demand and become the important alternatives of traditional power sources $[3,4,6]$.

Among a variety of renewable energy sources, PV is predicted to have the biggest generation, up to $60 \%$ of the total energy by the end of this century $[7,8]$, because the energy which is converted into electrical energy is the light from the sun, which is free, available almost everywhere and will still be present for millions of years long after all non-renewable energy sources have been depleted $[7,9]$. Though the PV module is still expensive, due to the large-scale manufacturing it is becoming increasingly cheaper in the last few years. In addition, the PV module has no moving parts, which have made it a very robust, long lifetime and low maintenance device. Based on the newest report of International Energy Agency (IEA) on installed PV power, the milestone of $100 \mathrm{GW}$ PV system all over the world was achieved at the end of 2012, and increased to $140 \mathrm{GW}$ at the end of 2013 which is shown in Fig. 1 [10].

Fig. 2 shows the share of grid-tied and off-grid PV installation. It can be seen that the off-grid market can hardly be compared with the grid-tied market. The evaluation of the share of grid-tied PV market per region from 2000 to 2013 is shown in Fig. 3. Though Asia started to dominate the market in the early 2000, after 2004 a great development can be seen in Europe. While Europe and Asia presented a major part of grid-tied PV installation globally in 2013, the Middle East \& Africa started to grow in 2012 and 2013. However, for grid-tied PV system, the power electronic technology plays an important role in the integration of PV energy sources

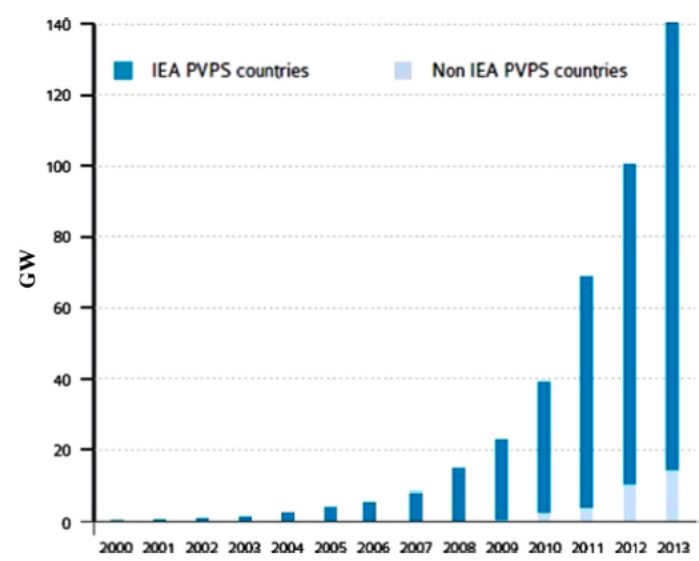

Fig. 1. Evaluation of PV installation [10].

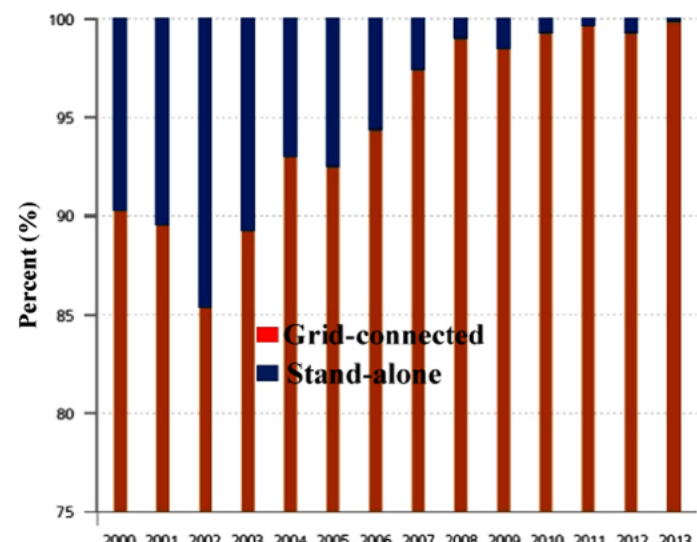

$2000200120022003200420052006200720082009201020112012 \quad 2013$

Fig. 2. Share of grid-connected and standalone PV installation [10]. 


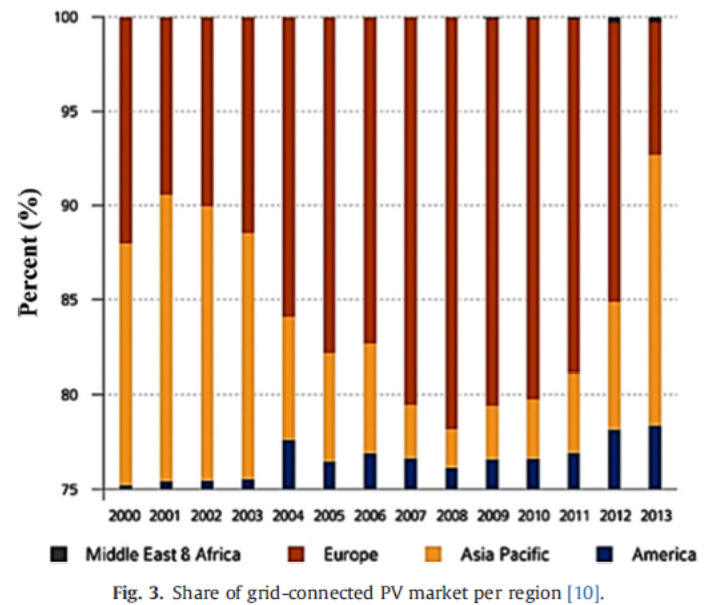

into the utility grid. Generally, a single-phase power converter is used to process the power of PV energy sources with certain matters like efficiency and cost as the main factors.

The scope of this paper is to provide an overview and discuss some trends in grid-tied photovoltaic inverter. Firstly, the overview of grid-tied PV system is presented including different types of PV inverter and the examination of demands for the utility grid, and the PV modules. Further, the leakage current issues of transformerless inverter for grid-tied PV system are discussed. Next, a review of transformerless topologies for grid-tied PV system by emphasizing the leakage current and efficiency is presented. At last, simulations are carried out of different topologies to compare their performance. Finally, the approaches are further discussed and evaluated to select the most suitable topologies for future grid-tied PV system.

\section{Grid-tied photovoltaic inverter}

\subsection{Classification of grid-tied PV inverter}

The general classifications of grid-tied PV inverters are as follows [11-15]:

- Central inverter;

- String inverter;

- Module integrated inverter;

- Multi-string inverters.

\subsubsection{Central inverters}

The central inverter system depicted in Fig. 4(a) was implemented in the past technology [15], where PV plants bigger than $10 \mathrm{kWp}$ were prepared in series or parallel strings and connected to the inverter. The inverters were mostly connected to a threephase application; thus no decoupling was necessary. The voltage generated by the series connected modules was high enough to satisfy the input voltage condition of the inverter [12]. However this inverter had some significant disadvantages such as high power losses due to centralized maximum power point tracking (MPPT), high voltage DC cables between PV panels and inverters due to high input voltage, non-flexible design, losses in the stringdiodes, and being expensive. Consequently, it was not possible to acquire the advantages of huge production. Furthermore, the power delivered to the grid by the central inverter was of very poor quality involving many current harmonics.

\subsubsection{String inverter}

The string inverter that offers a number of advantages are leading the present technology [15]. Fig. 4(b) describes the string inverter system, where a single PV string made by series connected solar panels is coupled to an inverter. The string voltage may be sufficient, and thus voltage boosting is not required. The normal operating voltage of string inverter is 340-510 VDC for 230 VAC application. The opportunity of using less PV modules connected in series is also available if a DC-DC booster or a line frequency transformer has been used. Compared to the central inverter, the string inverter has several advantages such as no string diode losses, individual MPPTs can be applied for every string, lower price due to huge production, and overall higher efficiency $[13,14]$.

\subsubsection{Module integrated inverter}

The module integrated inverter system is shown in Fig. 4(c), where an AC module made by a single solar panel and its own inverter is connected to the grid. There is no mismatch between the PV modules; as a result, the power loss is well minimized. It is also possible to obtain maximum power from the PV module as a result of its own inverter and MPPT [11]. The advantage of an easy expanding of the system is available here due to the modular structure. The main disadvantage is the reduced overall efficiency because of higher voltage amplification and installation cost. However, this can be overcome by huge production, leading to low manufacturing and retail costs.

\subsubsection{Multi-string inverter}

The multi-string inverter is the evaluation of string inverter depicted in Fig. 4(d), where each string made of several solar panels is coupled to its own DC-to-DC converter with individual MPPT and feed energy to a common DC to AC inverter. Consequently, each PV power plant with a few modules can be functioned separately. The advantages of string and module integrated inverter is combined here. Since each PV string is controlled individually, the overall efficiency is higher. There are several advantages of multi-string inverters such as cost reduction, more flexible, small DC-link capacitor, and high energy reveal due to local MPP tracking and optimum monitoring of the PV system $[12,16]$.

\section{Design of grid-tied PV inverter}

The PV generates DC voltage; thus, it requires a converter to convert into a voltage of corresponding amplitude at the main frequency for feeding it into utility grid as shown in Fig. 5. Most of the countries report installing PV system by counting DC power, but some report AC power also. The inverter role in grid-tied PV system is to be the interface between two energy sources: the PV module on one side and the utility grid on the other side. Since the inverter converts DC power of PV module into AC power for feeding it into utility grid, it is responsible for power quality that needs to be satisfied by the requirement of different standards, which are briefly described in the next section. Depending on the galvanic isolation between the PV module and the grid, the gridtied PV inverter can be categorized as isolated or non-isolated. The galvanic isolation between the PV module and the grid can be observed by using a line frequency transformer or a high frequency transformer that adjusts converter DC voltage $[6,17,18]$. Due to size, weight and price in favor of high frequency transformers, the tendency of removing the line frequency transformers is 
a

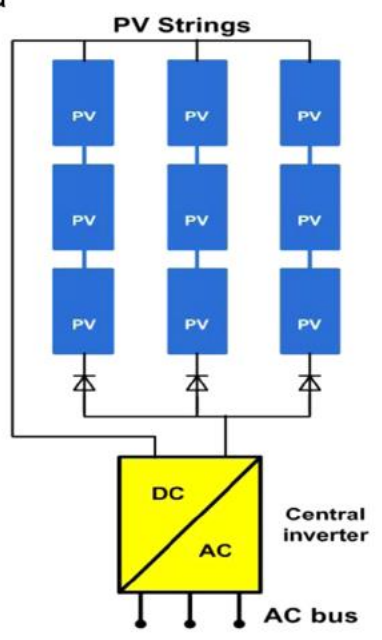

C

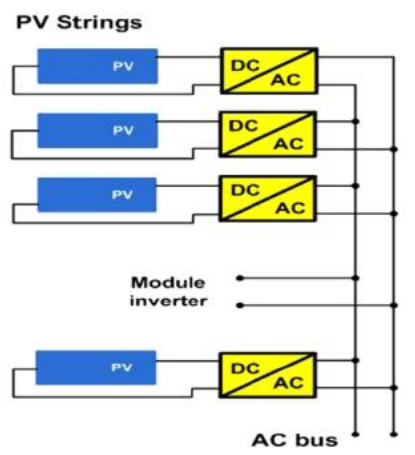

b

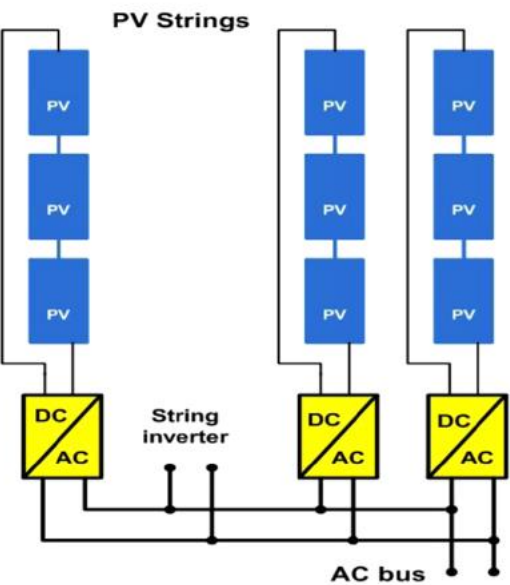

d

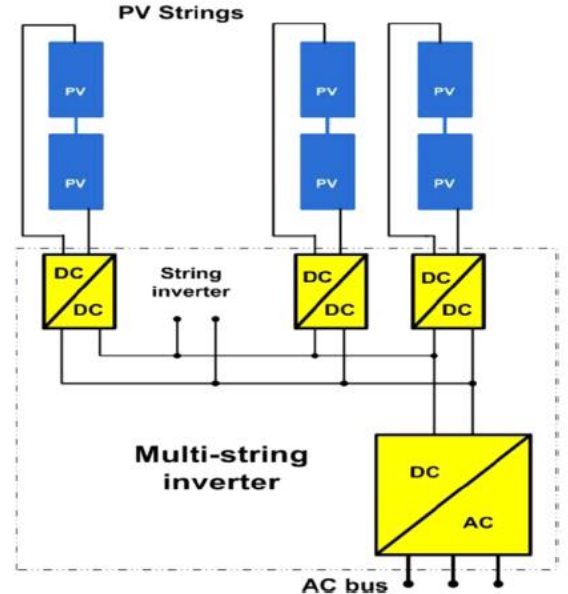

Fig. 4. Different types of grid-tied PV inverter: (a) central inverter; (b) string inverter; (c) module inverter; and (d) multi-string inverter.

increased when designing the new converter. Also the existence of high-frequency transformer requires several power stages, which makes reducing cost and increasing efficiency a challenging task [19-21]. On the other hand, transformerless grid-tied inverter has the benefits of lower cost, higher efficiency, smaller size, and weight when compared to the ones with transformer [22-28]. Furthermore, during the last few years, a change of paradigm concerning the size of the grid-tied inverter has been observed $[29,30]$. Because of some limitations of big central inverters of power more than $100 \mathrm{~kW}$, the present technology consists of the small size string or multi-string inverters which improve the MPPT of large PV groups of panel [14,31,32].

\section{Requirements of the grid-tied PV system}

The photovoltaic system connected to the grid involves two major tasks: (1) it must be ensured that the solar panels are operated at MPP; and (2) the injected current to the grid must be sinusoidal which has to comply with some specific standards. In this section, these tasks are further investigated.

\subsection{Requirements of the grid}

The grid connected PV system must comply with some specific standards that are regulated by the utility in each country such as IEEE 1547.1-2005, VDE0126-1-1, EN 50106, and IEC61727. These standard deal with matters like total harmonic distortion (THD and individual harmonic current levels, injected DC current level and leakage current, range of voltage and frequency for regular operation, power factor (PF), detection of islanding operation (islanding or non-islanding functions), grounding of the system, and automatic reconnection and synchronization [33-36]. Table 1 summarizes the requirements of IEEE 1547 standard [14,37].

As seen in Table 1, the IEEE 1547 standard set some restrictions on maximum acceptable DC current injection into the utility grid. 


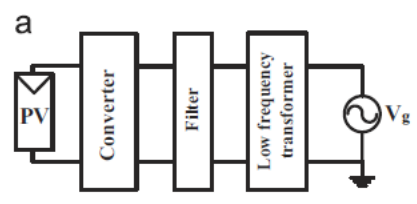

b

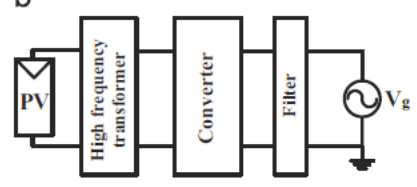

C

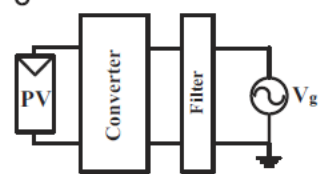

Fig. 5. Grid-tied PV system using (a) grid size low-frequency transformer; (b) DC side high-frequency transformer; and (c) transformerless inverter.

Table 1

IEEE 1547 requirements for grid connection [14].

\begin{tabular}{ll}
\hline Nominal power & $30 \mathrm{~kW}$ \\
Harmonic currents & $(2-10) 4.0 \%$ \\
& $(11-16) 2.0 \%$ \\
& $(17-22) 1.5 \%$ \\
& $(23-34) 0.6 \%$ \\
& $(>35) 0.3 \%$ \\
& THD $5 \%$ \\
& $<0.5 \%$ of rated output current \\
DC current injection & $V<50 \%$ or $V>137 \% 6$ cycles \\
Abnormal voltage & $50 \%<V<88 \%$ or $110 \%<V<137 \% 120$ cycles \\
disconnection & $f<$ rated $-0.7 \mathrm{~Hz} 6$ cycles \\
Abnormal frequency & $f>$ rated $+0.5 \mathrm{~Hz} 6$ cycles \\
disconnection & \\
\hline
\end{tabular}

Generally, the saturation of distribution transformer is neglected by limiting the DC current injection [38,39]. Nevertheless, the limit is quite small in IEEE 1547 standard ( $<0.5 \%$ of rated output current) and it is difficult to maintain this value precisely with the exciting circuit inside the inverter. Rather this can be suppressed by providing a galvanic isolation between PV module and the grid. However, this matter is a great concern in case of transformerless inverter and different control strategies have been investigated to effectively limit the DC current injection into the utility grid [40-42]. The DC current injection requirement given by different standards could be summarized as shown in Table 2 .

The EN 50106 standard deals with the voltage characteristics of electrical energy in the public distribution system. It defines the requirements of the main voltages and their permitted deviation ranges at the point of common coupling (PCC) in low voltage and medium voltage distribution system under normal operating condition. Table 3 shows the requirement of the EN 50106 standard. In the table, low and medium voltages are defined as the phase-phase nominal root mean square (RMS) voltage that does not exceed $1000 \mathrm{~V}$ for low voltage and lies between 1 and $35 \mathrm{kV}$ for medium voltage.

The German VDE-0126-1-1 standard is the only standard that specifically deals with transformerless PV inverter concerning fault or leakage current levels. According to VDE-0126-1-1 standard, the disconnection time of the inverter from the grid is $0.3 \mathrm{~s}$ when the RMS value of leakage current is greater than $30 \mathrm{~mA}$. The RMS

Table 2

Maximum DC current injection described in different standard.

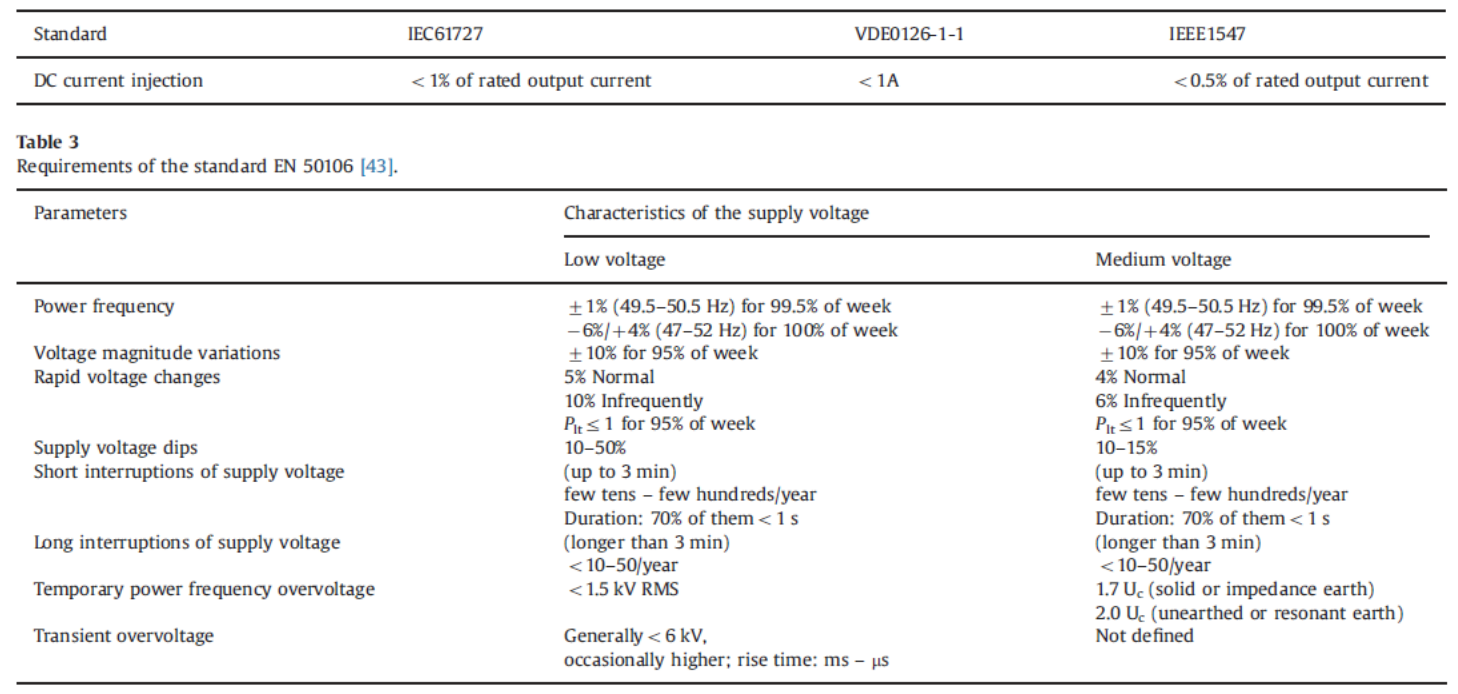

\section{Link to Full-Text Articles :}

